\title{
A Case of Diabetic Ketoacidosis in Pregnancy
}

\section{Kristine Gayle T Suarez, and Gerry H Tan*}

Division of Endocrinology, Diabetes and Metabolism, Department of Internal Medicine, Cebu Doctors University, College of Medicine Cebu Doctors University Hospital, Cebu, Philippines

*Corresponding author: Gerry H Tan, Division of Endocrinology, Diabetes and Metabolism, Department of Internal Medicine, Cebu Doctors University, College of Medicine Cebu Doctors University Hospital, Cebu, Philippines, Tel: +63-32-4124803; E-mail: endoking2@yahoo.com.ph

Received: 11 Nov, 2019 | Accepted: 04 Dec, 2019 | Published: 10 Dec, 2019

Citation: Suarez KGT, Tan GH (2019) A Case of Diabetic Ketoacidosis in Pregnancy. J Diab Res Ther 5(3): dx.doi.org/10.16966/2380-5544.149

Copyright: (C) 2019 Suarez KGT, et al. This is an open-access article distributed under the terms of the Creative Commons Attribution License, which permits unrestricted use, distribution, and reproduction in any medium, provided the original author and source are credited.

\section{Abstract}

Objective: Diabetes has affected people worldwide across different ethnicity, gender, including the obstetric population. We report a case of a 28 year-old, multi-gravid, which has not been previously, diagnosed with diabetes in her $1^{\text {st }}$ and $2^{\text {nd }}$ pregnancy with no family history of diabetes, but developed severe Diabetic Ketoacidosis (DKA) in her third pregnancy.

Case presentation: A 28 year old, Filipino, known diabetic on insulin therapy on her $24^{\text {th }}$ week age of gestation presented with dyspnea and vomiting. On admission, she was received lethargic and hypotensive with a blood pressure of 80/50 mmhg, tachycardic and tachypneic. She had muscle weakness and significant premature uterine contractions. Her random blood glucose was only $357 \mathrm{mg} / \mathrm{dl}(9.2 \mathrm{mmol} / \mathrm{L}) \mathrm{but}$ was severely acidotic. Arterial Blood Gas revealed a ph of 7.036, $\mathrm{HCO}_{3}$ of $2.6 \mathrm{mmol} / \mathrm{L}$ and $\mathrm{PCO}_{2}$ of $9.5 \mathrm{mmHg}$, with serum ketones of $4.9 \mathrm{mmol} / \mathrm{L}$ and an anion gap of $34 \mathrm{mmol} / \mathrm{L}$. An ultrasound of the abdomen revealed a single live, 24 weeks by fetal biometry with good cardiac and somatic activity, but with sonographic consideration of hydrops fetalis with cardiac enlargement. She was advised to have a complete prenatal diagnostic work up for which she did not comply, and came in with a critical presentation that has lead to maternal and fetal complications.

Discussion: Pregnancy is a physiologic state that can lead to a significant change in metabolism. Understanding the biochemical feedback compared to the non-pregnant state is important in the diagnosis of diabetes. A serious but rare metabolic complication is DKA in Pregnancy.

Our case is a rare complication of diabetes in pregnancy in the era of approved insulin analogue use. The incidence of DKA in Pregnancy range from $0.5 \%$, the lowest accounted rate in western countries to $8.9 \%$ in a study conducted in China. The degree of hyperglycemia in pregnancy complicated by DKA does not present with significant elevation as it may be blunted due to several maternal and fetal adaptive mechanisms. Therefore, it is a diagnostic challenge because the glucose levels are either near normal or not as elevated as the non-pregnant DKA state due to the biochemical feedback.

Conclusion: This case illustrates a rare but preventable complication of pregnancy. If diagnosed right away and managed properly, maternal complications can be minimized. Review of hospital records show no similar reported case in the files of Cebu Doctors' University Hospital. Our case illustrates vigilance in diagnosis as it may have long term complications both for the mother and the unborn child.

Keywords: Diabetic ketoacidosis; Diabetic ketoacidosis in pregnancy; Diabetes mellitus

\section{Background}

Diabetes in Asian countries including the Philippines features an alarming drastic increase. It is predicted that Asia will witness the greatest number of people with diabetes by 2025 [1]. It has affected people worldwide across different ethnicity, gender, including the obstetric population.

Pregnancy is a time of wonder and amazement but it also involves a physiologic state that increases counter regulatory hormones, and causes a significant change in metabolism. One of the serious but very rare metabolic complications is Diabetic Ketoacidosis noted in pregnancy (DKA). The incidence of DKA in pregnancy range from $0.5 \%$, the lowest accounted rate in western countries to $8.9 \%$ in a study conducted in China [2]. Tight glycemic control is important as poor control can lead to detrimental effects not only to the mother but also to the fetus. In early pregnancy, it increases the risk of spontaneous abortion and congenital malformation, while late in pregnancy can result in preterm labor, stillbirth and macrosomia.

Diabetic Ketoacidosis (DKA) is a life threatening medical emergency with high mortality if undetected. It occurs when the body is unable to use glucose because of defects in insulin function and production, and undergoes ketogenesis. DKA in Pregnancy is a rare occurrence but a serious complication that brings several challenges in the diagnosis, management and prevention [3]. It brings a high maternal and fetal mortality with rates as high as $30 \%$ to $90 \%$, and requires treatment, prompt recognition and resuscitative therapy to improve medical and obstetric outcomes $[4,5]$. 
The state of serum ketones has been estimated to be two to four times greater than in the non-pregnant state [4]. There is a simultaneous increase in counter regulatory hormones that cause significant modification in metabolism, such as, lipolysis and proteolysis. These augment gluconeogenesis and glycogenolysis, contributing to the development of hyperglycemia. Given these physiologic and metabolic changes in pregnancy, the clinical presentation of DKA stays the same as in non-pregnant women, but diagnosis is often delayed due to the non-classical presentation of the biochemical abnormality presenting with much lower blood sugar level that is not seen in true DKA cases in non-gravid diabetics. Early recognition of the case and a timely diagnosis are critical for prompt initiation of treatment to avoid maternal and fetal complications.

\section{Case Presentation}

This is a case of a 28 year old woman who developed Diabetic Ketoacidosis on her $24^{\text {th }}$ week age of gestation $(\mathrm{AOG})$ and presented with dyspnea, drowsiness, and vomiting at the Emergency Department (ED).

She has no known family history and no prior history of Diabetes Mellitus at Gravida 1, 2010, and Gravida 2, 2011. She delivered a live female and male neonate, respectively, through Normal Vaginal Delivery. In 2015, four years after the delivery of the second child, patient was diagnosed to have hyperglycemia and was treated as a case of Type $1 \mathrm{DM}$ and maintained on subcutaneous insulin therapy of NPH Insulin 20 units once a day. However due to financial reasons, she was not compliant to her medical nutrition therapy, insulin medications nor home glucose monitoring.

At 8 weeks AOG of the present pregnancy, she had her first prenatal check-up at a local health station, and was advised to visit a tertiary hospital for a complete diagnostic work up. However neither blood tests nor any hospital visits were made until her 21 week AOG, where an ultrasound was done. Apparently due to non-compliance to her insulin medication, she had blood sugars that were in the range of 300 to $500 \mathrm{mg} / \mathrm{dl}(7.7-13 \mathrm{mmol} / \mathrm{L})$.

Few days prior to admission, she apparently missed her insulin requirements for the past 5 days for personal and financial reasons. Subsequently, she started to feel tired and sleepy with increased occurrence of polydipsia and polyuria until the day of admission where she was rushed with a critical clinical presentation. She was received afebrile, drowsy, hypotensive with a blood pressure of $80 / 50$ $\mathrm{mmHg}$, tachycardic and tachypneic. She had 7 episodes of vomiting, complained of marked fatigue, muscle weakness and was noted to have premature uterine contractions. Her physical examination was significant for cachexia, dry mucosa and exhibited deep breathing with a fruity breath odor.

Her random capillary blood glucose was $357 \mathrm{mg} / \mathrm{dl}(9.2 \mathrm{mmol} / \mathrm{L})$, with positive serum ketones of 4.9, and an Arterial Blood Gas (ABG) revealed, $\mathrm{pH}$ of $7.036, \mathrm{HCO}_{3}$ of $2.6 \mathrm{mmol} / \mathrm{L}$ and $\mathrm{PCO}_{2}$ of $9.5 \mathrm{mmHg}$, consistent with primary metabolic acidosis. Further evaluation revealed the presence of high Anion Gap (AG) of $34 \mathrm{mmol} / \mathrm{L}$. The biochemical evaluation confirmed a diagnosis of DKA in the setting of Pregnancy.

She was given fluid hydration followed by intravenous insulin bolus of 10 units. An insulin drip was then initiated with an infusion rate of 0.1 units $/ \mathrm{kg} /$ hour. Hourly monitoring of blood sugar was done until then normalization of the anion gap. With the eventual improvement of anion gap of $22 \mathrm{mmol} / \mathrm{L}$ in less than 8 hours, patient was then started on multiple daily insulin regimens, with basal insulin given at least one hour prior to discontinuation of the insulin drip. She was noted to have improvement of sensorium becoming more awake, responsive and oriented with stable vital signs [6,7].

She was subsequently discharged with a normal AG and normal $\mathrm{pH}$ with capillary blood sugar averaging between $85-160 \mathrm{mg} / \mathrm{dl}$ (2-4 $\mathrm{mmol} / \mathrm{L}$ ). Due to the possible harmful effects of DKA in her present pregnancy, assessment of the patient was done clinically with daily fetal movement and monitoring of fetal heart sounds. To rule out any abnormality, an ultrasound of the abdomen was done which revealed a single live intrauterine pregnancy, cephalic presentation, 24 weeks by fetal biometry with good cardiac and somatic activity. There was also an adequate amniotic fluid volume, but with sonographic consideration of hydrops fetalis with cardiac enlargement.

The patient was sent home and given dietary instructions, with strict home glucose monitoring recommendations and insulin regimen instructions. She was instructed to have regular followed up as outpatient with his local physician and fetal monitoring with her local obstetrician $[3,8,9]$.

\section{Discussion}

DKA results from a relative or total insulin deficiency combined with excess counter regulatory hormones. In pregnancy, these counter regulatory hormones are amplified. Both insulin deficiency and excess of glucose can lead to the development of DKA [10]. The reduced levels of insulin in proportion to glucose would enhance gluconeogenesis, glycogenolysis and ketone body formation. Ketosis results from a striking increase in the recruitment of free fatty acid from adipocytes those results to the shift of energy substrate favoring ketone body synthesis, instead of being metabolized to triglycerides and very low density lipoprotein $[10,11]$.

In a gravid state, there is a related physiologic change that predisposes a pregnant woman with diabetes to develop DKA. Pregnancy apparently is a state of respiratory alkalosis where a compensatory mechanism drops the bicarbonate levels and affects the buffering system causing the pregnant woman to be acidotic and predisposing to develop DKA [3]. There is also a relative insulin resistance in pregnancy due to changes in carbohydrate and fat metabolism with enhanced lipolysis and elevated free fatty acids [5,11]. Another factor that contributes to insulin resistance includes the hormonal change associated during late pregnancy such as placental lactogen, increased cortisol, and progesterone. Pregnancy is also considered to be an increased state of starvation, such that fasting overnight, can accelerate lipolysis, and ketone body concentrations that are twice or four times higher than the non-pregnant state $[5,8,10,11]$. Under these conditions, DKA can develop more rapidly and at lower serum concentrations of glucose [11].

The patient presented with change in sensorium, tachypnea and hypotension in a setting of pregnancy. Patient was referred from her local district in the province of Cebu and therefore no information was retrieved regarding her past medical history except for the normal pregnancies and deliveries in the first two pregnancies. On admission, random blood sugar of $357 \mathrm{mg} / \mathrm{dl}$ was recorded but not high enough to be considered in the range of a hyperglycemic emergency. The presence of lethargy, tachypnea, and ketotic breath led to the biochemical evaluation that showed positive ketones, metabolic acidosis and a high anion gap consistent with DKA.

The likely mechanism of DKA in Pregnancy presenting glucose levels that may not be as high as the non-pregnant DKA state includes the accelerated glucose usage of fetoplacental component $[2,4]$. There 
is also an increased glycosuria, due to increased renal blood flow thus enhancing glomerular filtration without an analogous increase in tubular glucose reabsorption [2-4]. Other factors to explain include an enhanced maternal use of glucose due to estrogen and progesterone and its dilutional consequence from an increased plasma volume in pregnancy.

Although with a different physiologic response in pregnancy, the manifestations of DKA are the same in the non-pregnant state. They usually present with nausea, vomiting, polyuria or polydypsia, abdominal pain, muscle weakness, shortness of breath, tachycardia, hypotension, tachypnea or kussmaul's respiration, and drowsiness which may develop to coma.

Hyperglycemia and hyperosmolarity as a consequence, results in osmotic diuresis leading to depletion of intravascular volume thus, producing poor tissue perfusion, and diminished cardiac and renal function. Maternal complications would ensue, such as, acute renal failure, adult respiratory distress syndrome, cerebral edema, coma and even, death $[2-4,8]$. During DKA in Pregnancy, the fetal brain is vulnerable to increased 3 beta hydroxyl butyrate and lactate concentrations, leading to a reduction in glucose ensuing to brain injury $[3,8]$. The decision to deliver should be individualized based on evaluation of maternal status and fetal gestational age. Our patient did not have ante partum evaluation to diagnose poor hyperglycemic state. As such, patient was not counseled regarding possible complication if diabetes went uncontrolled. Likewise, fetal evaluation during the admission did not warrant immediate delivery of the baby.

Prevention strategies should have been assumed before conception to avert DKA. Woman with diabetes should execute planning and should receive facts regarding the risks associated with uncontrolled blood glucose in pregnancy. Diabetes education is key from the time a diagnosis of diabetes is made to the time the woman decides to marry and have a family. Awareness of the disease and its complications can reduce of fetal and maternal risk associated during pregnancy [3]. Preconception counseling making sure blood sugar is tightly controlled with frequent home glucose monitoring as well as constant and periodic visits to the diabetes specialist is of paramount importance. During pregnancy, routine screening is important to all obstetric population to avoid any fetal malformation. Adjustment of insulin dose, dietary pattern counseling and monitoring of physical activity should be made. She should be educated that missing insulin doses resulting in poor blood glucose control is one important precipitating factor of DKA in pregnancy $[9,12]$.

This case illustrates a rare and preventable complication of pregnancy. Our patient is the first ever reported case of Diabetic Ketoacidosis in Pregnancy in our hospital. Once a diagnosis of DKA in Pregnancy is made, it is important to carefully monitor the wellbeing of the fetus with the help of our obstetric colleagues and stabilize the maternal metabolic state without any immediate plan for fetal delivery. It is important to emphasize that education and awareness of the disease and its complications are necessary to avoid any recurrence of DKA in subsequent Pregnancies [13,14].

\section{Conclusion}

Our case of Diabetic Ketoacidosis in pregnancy is unique because this is a case of a diabetic emergency where the presentation is unusual. The degree of hyperglycemia in pregnancy complicated by DKA does not present with a significant elevation of blood sugar as it may be blunted by several maternal and fetal adaptive mechanisms that are not present in a non-pregnant state. Diabetic ketoacidosis can easily be aggravated by the changes in both the physiologic and biochemical processes that are distinct in pregnancy.

As DKA is a catastrophic event during pregnancy, complicating almost nine percent of diabetic pregnant women that can result in both maternal and fetal mortality, early recognition and strong suspicion are keys to proper diagnosis and management, so effective planning strategies can be made.

\section{Acknowledgment}

Paper was presented as a poster at the American Association of Clinical Endocrinologists Annual Meeting April 2018 in Boston Massachusetts, USA.

Supported in Part by AACE Philippines Research Advocacy.

\section{References}

1. Tan GH (2015) Diabetes Care in the Philippines. Annals of Global Health 81: 1-2.

2. Baagar KA, Aboudi AK, Khaldi HM, Alowinati BI, Abou-Samra AB, et al. (2017) Retrospective Analysis of Diabetic Ketoacidosis in Pregnant Women over a period of 3 years. Endocrinology and Metabolic Syndrome 6: 1-8.

3. Mohan M, Baagar KAM, Lindow S (2017) Management of diabetic ketoacidosis in pregnancy. The obstetrics and Gynecologist 19: 5562.

4. Chico M, Levine SN, Lewis DF (2008) Normoglycemic diabetic ketoacidosis in pregnancy. Journal of Perinatology 28: 310-312.

5. Channawar S, Prasad M, Jain N, Chauhan AR (2014) Successful management of Diabetic Ketoacidosis. Journal of Post Graduate Gynecology and Obstetrics.

6. Kasper DL, Fauci AS, Hauser SL, Longo DL, Jameson JL, et al. (2018) Harrison's Principles of Internal Medicine. $20^{\text {th }}$ Edition, McGrawHill 2869-2872.

7. de Veciana Margarita (2013) Diabetic Ketoacidosis in Pregnancy. Semin Perinatol 37: 267-273.

8. Kamalakannan D, Baskar V, Barton DM, Abdu TAM (2003) Diabetic Ketoacidosis in Pregnancy. Postgrad Med J 79: 454-457.

9. Himuro H, Sugiyama T, Nishigori H, Saito M, Nagase S, et al. (2014) A case of a woman with late pregnancy onset DKA who had a normal glucose tolerance in the first trimester. Endocrinol Diabetes Metab Case Rep 2014: 130085.

10. Schneider MB, Umpierrez GE, Ramsey RD, Mabie WC, Bennett KA (2003) Pregnancy Complicated by Diabetic Ketoacidosis. Diabetes Care 26: 958-959.

11. Papadakis MA, McPhee SJ, Rabow MW (2017) Current Medical Diagnosis and Treatment. $6^{\text {th }}$ Edition, McGraw hill, USA 1245-1249.

12. American Diabetes Association (2015) Classification and Diagnosis of Diabetes. Diabetes Care 38: S8-S16.

13. Kitabchi AE, Umpierrez GE, Miles JM, Fisher JN (2009) Hyperglycemic Crises in Adult patients with Diabetes. Diabetes Care 32: 1335-1343.

14. Mandal A (2017) History of Diabetes. 